\title{
Paradigm shifts in the medical and surgical management of rhinologic and allergic disease
}

$\mathbf{I}^{\mathrm{t}}$ is such an honor to have the opportunity to return as Guest Editor for this edition of the American Journal of Rhinology and Allergy (AJRA), and I want to express my deepest gratitude to the Editors-in-Chief Rakesh Chandra, Raj Sindwani, and Anju Peters for their ongoing support and outstanding leadership. The AJRA is committed to presenting the latest and highest-quality clinical and translational research in the fields of rhinology, allergy, and skull base surgery. To that end, the November-December issue of the AJRA encompasses a broad spectrum of studies that offer new insights into the treatment of rhinologic and allergic disease.

Chronic rhinosinusitis (CRS) has been characterized as a complex, inflammatory disorder with environmental, genetic, and local host factors, all contributing to its pathogenesis. ${ }^{1}$ However, the precise etiology of CRS is still unknown, and its optimal medical management remains controversial. ${ }^{2}$ In this issue of the AJRA, several studies aim to better our understanding of the mechanism of action of various medical therapies currently available for CRS. Because Th2 inflammation is known to play a role in certain CRS subtypes, Kennedy et al. ${ }^{3}$ investigated the impact of itraconazole on the differentiation of naive $\mathrm{T}$ cells into Th2 like cells in vitro.

No effect on proliferation of unbiased $\mathrm{T}$ cells was evident with exposure to varying concentrations of itraconazole, which may explain, at least in part, why recent clinical trials have reported no clinical benefit with the use of antifungal therapy for CRS.4,5 In contrast, Shin et al. ${ }^{6}$ demonstrated that doxycycline successfully inhibited transforming growth factor $\beta 1$-induced matrix metalloproteinase 2 expression, migration, and collagen contraction in nasal polyp-derived fibroblasts. Such findings underscore the effectiveness of doxycycline in reducing nasal polyp size in patients with CRS with nasal polyposis. ${ }^{7}$ Similarly, Ko et al., ${ }^{8}$ showed that rapamycin inhibited transforming growth factor $\beta 1$-induced myofibroblast differentiation, extracellular matrix production, and collagen contraction in nasal polyp-derived fibroblasts. ${ }^{8}$ Therefore, rapamycin may also become a part of our treatment armamentarium for CRS with nasal polyposis in the future.

Alterations in the nascent sinus microbiome have recently been postulated to contribute to development of CRS. ${ }^{9}$ Consequently, current research has focused on the potential therapeutic effects of topical probiotics and/or antibiotics in restoring resident microbiota and modulating immune responses. In their in vitro study, Schwartz et al. ${ }^{10}$ found that gram-positive probiotics induced secretion of IL10 (which downregulates host inflammatory reactions) without damaging sinus epithelia. Likewise, in their clinical study, Lee and Davis ${ }^{11}$ assessed the efficacy of culture-directed topical antibiotic irrigations in the treatment of 58 patients with recalcitrant CRS. Improvement in LundKennedy endoscopic $(p=0.05)$ and 20-item Sino-Nasal Outcome Test ( $p=0.16)$ scores were observed after 1 month of therapy, with $72 \%$ of the patients having negative posttreatment cultures. ${ }^{11}$ Although promising, randomized controlled trials are still necessary to ade- quately evaluate the role of topical probiotics and/or antibiotics in refractory CRS.

In addition to CRS, this issue of the AJRA also includes studies that investigated the epidemiology and management of allergic disease. In a study of $>17,500$ subjects, Kim et al. ${ }^{12}$ determined that dry eye was significantly associated with allergic rhinitis and house-dust mite (Dermatophagoides farinae) sensitization in the Korean adult population. Following this tangent, Krajewska-Wojtys et al. ${ }^{13}$ discovered that local allergic rhinitis was underdiagnosed in young patients (12-18 years). In their series of 121 patients, $42.5 \%$ (73) with a previous diagnosis of nonallergic rhinitis were ultimately found to have positive reactions to grass (Phleum partense), Artemisia, and birch pollen based on skin-prick testing and serum specific IgE measurements. Furthermore, in the study by Kaur et al., ${ }^{14}$ innate immune genes analysis of middle ear fluid samples revealed differential expression in children who were otitis prone versus non otitis prone children.

With respect to treatment, Greiwe and Bernstein ${ }^{15}$ highlighted the advantages of combination therapy (i.e., intranasal corticosteroids-topical decongestants; azelastine-fluticasone) in management of complex rhinitis phenotypes. In addition, Arcigil et al. ${ }^{16}$ performed a literature review to explore new routes for administration of allergen immunotherapy. Intralymphatic (direct injection of allergens into lymph nodes), epicutaneous (placement of allergen patches on the skin), nasal (spraying of allergens into the nasal cavity), and oral cavity and/or mucosal delivery were all found to be safe and effective. Such novel application methods may represent new alternatives to subcutaneous and sublingual immunotherapy in the future. In another systematic review, Kennedy et al. ${ }^{17}$ discussed the pathogenesis, risks-benefits of aspirin desensitization, and the role of surgical intervention in aspirin-exacerbated respiratory disease.

In terms of olfaction, Bilici et al. ${ }^{18}$ analyzed the effects of radioactive iodine treatment for papillary thyroid carcinoma on smell function. In a prospective clinical study of 62 patients, $46 \%$ experienced olfactory deterioration, with significant reduction in threshold, identification, and total smell scores (Connecticut Chemosensory Clinical Research Center olfactory test) up to 1 year after RAI. In addition, Kohli et al. ${ }^{19}$ examined the relationship between olfactory cleft opacification and olfaction in CRS patients with (12) and without (26) nasal polyposis. Volumetric analysis of the olfactory cleft using computed tomography imaging revealed significant correlations between the degree of opacification and olfactory threshold, discrimination, and identification scores (Sniffin' Sticks test), findings that were more pronounced in the CRS with nasal polyposis group. Thus, olfactory cleft opacification on computed tomography imaging may be associated with clinical olfactory dysfunction.

With continued advances in endoscopic techniques and instrumentation, new surgical approaches have been developed for the man- 
agement of rhinologic and skull base pathology. Massey et al. ${ }^{20}$ described the novel use of an ultrasonic bone aspirator for extended endoscopic frontal sinusotomy (Draf IIb or Draf III) in anterior skull base surgery. In a case series of 18 patients, no differences in frontal ostial patency, Lund-Mackay scores, or revision surgery rates were observed between those who underwent Draf IIb/III sinusotomies with an ultrasonic bone aspirator versus conventional instrumentation. In their series, Kuan et al..$^{21}$ explored the clinical significance of retromaxillary cells in revision endoscopic sinus surgery. Incompletely dissected retromaxillary cells were seen in 73\% (101/138 sides) despite evidence of previous posterior ethmoidectomies, with $65 \%$ cells harboring residual disease. Such findings indicated that inadequate dissection of unrecognized retromaxillary cells may contribute to recalcitrant CRS and the need for revision ESS. Likewise, Hong et al. 22 performed a systematic review to assess the efficacy and safety of open versus endoscopic septoplasty. Pooled data from 14 studies showed greater improvement in postoperative symptoms and fewer complications with the endoscopic technique.

In a survey of the American Rhinologic Society examining pediatric CRS, Beswick et al. ${ }^{23}$ found that adenoidectomy was included by $90 \%$ of respondents as part of first-line surgical treatment and endoscopic sinus surgery by $85 \%$ for patients who failed primary surgical intervention. Interestingly, balloon catheter dilation was uncommonly used, with a majority of respondents $(66 \%)$ rarely or never using the technology for pediatric CRS. Nevertheless, additional research is necessary to determine whether such practice patterns are representative of the general otolaryngology community as a whole or whether the aforementioned findings would change if a different target group were surveyed (i.e., pediatric otolaryngologists.)

With paradigm shifts in medical and surgical treatment, postoperative management of endoscopic sinus and/or skull base surgery has also evolved. Traditionally, lumbar drains have been used after endoscopic cerebrospinal fluid leak repair to facilitate healing and reduce the risk of recurrence. ${ }^{20}$ However, recent studies have challenged this approach. In their series of 107 patients, Adams et al. ${ }^{24}$ found no difference in cerebrospinal fluid leak recurrence rates between those with (19) and without (88) lumbar drains, irrespective of etiology. Repair technique and defect size (as opposed to body mass index) were the only reported predictors of recurrence. Likewise, D'Anza et al. ${ }^{25}$ conducted a comprehensive literature review to examine the role of lumbar drains in endoscopic resection of skull base tumors. A meta-analysis of three studies showed no statistically significant difference in outcomes between patients with and without lumbar drains, similar to what was observed in the series by Adams et al. ${ }^{24}$

Thus, this edition of the AJRA comprises a potpourri of articles that offer new insights into treatment paradigms for allergic and rhinologic disease. We hope that our readership finds the content of this issue to be informative not only from a clinical perspective but also from an academic standpoint to incite further innovations in rhinology research in the future.

\section{REFERENCES}

1. Kennedy DW. Pathogenesis of chronic rhinosinusitis. Ann Otol Rhinol Laryngol 193:6-9, 2004.

2. Lee JT, and Kennedy DW. Endoscopic sinus surgery. In Head \& Neck Surgery-Otolaryngology, 4th ed. Bailey BJ, and Johnson JT (Eds). New York: Lippincott-Raven 459-475, 2006.

3. Kennedy J, Steinke J, Liu L, et al. Failure of itraconazole to prevent Th2 immune deviation: Implications for chronic rhinosinusitis. Am J Rhinol Allergy 30:379-384, 2016.
4. Ebbens FA, Scadding GK, Badia L, et al. Amphotericin B nasal lavages: Not a solution for patients with chronic rhinosinusitis. J Allergy Clin Immunol 118:1149-1156, 2006.

5. Liang KL, Su MC, Shiao JY, et al. Amphotericin B irrigation for the treatment of chronic rhinosinusitis without nasal polyps: A randomized, placebo-controlled, double-blind study. Am J Rhinol 22:52-58, 2008.

6. Shin J, Park J, Lee S, et al. Effect of doxycycline on transforming growth factor-beta-1-induced matrix metalloproteinase 2 expression, migration, and collagen contraction in nasal polyp-derived fibroblasts. Am J Rhinol Allergy 30:385-390, 2016

7. Van Zele T, Gevaert P, Holtappels G, et al. Oral steroids and doxycycline: Two different approaches to treat nasal polyps. J Allergy Clinical Immunol 125:1069-1076.e4, 2010.

8. Ko D, Shin J, Um J, et al. Rapamycin inhibits transforming growth factor beta 1 induced myofibroblast differentiation via the phosphorylated-phosphatidylinositol 3-kinase mammalian target of rapamycin signal pathways in nasal polyp-derived fibroblasts. Am J Rhinol Allergy 30:e211-e217, 2016.

9. Lee JT, Frank DN, and Ramakrishnan V. Microbiome of the paranasal sinuses: Update and literature review. Am J Rhinol Allergy 30:3-16, 2016.

10. Schwartz JS, Peres A, Endam L, et al. Topical probiotics as a therapeutic alternative for chronic rhinosinusitis: A preclinical proof of concept. Am J Rhinol Allergy 30:e202-e205, 2016.

11. Lee V, and Davis G. Culture-directed topical antibiotic treatment for chronic rhinosinusitis. Am J Rhinol Allergy 30:414-417, 2016.

12. Kim M, Oh J, Park C, et al. Dry eye disease and allergic conditions: A Korean nationwide population-based study. Am J Rhinol Allergy 30:397-401, 2016

13. Krajewska-Wojtys A, Jarzab J, and Gawlik R. Local allergic rhinitis to pollens is underdiagnosed in young patients. Am J Rhinol Allergy 30:e198-e201, 2016.

14. Kaur R, Casey J, and Pichichero M. Differences in innate immune response gene regulation in the middle ear of otitis prone and nonotitis prone children. Am J Rhinol Allergy 30:e218-e223, 2016.

15. Greiwe J, and Bernstein J. Combination therapy in allergic rhinitis: What works and what doesn't. Am J Rhinol Allergy 30:391-396, 2016

16. Aricigil M, Muluk N, Sakarya E, et al. New routes of allergen immunotherapy. Am J Rhinol Allergy 30:e193-e197, 2016.

17. Kennedy J, Stoner A, and Borish L. Aspirin-exacerbated respiratory disease: Prevalence, diagnosis, treatment, and considerations for the future. Am J Rhinol Allergy 30:407-413, 2016.

18. Bilici S, Edizer D, Yigit O, et al. The effects of radioactive iodine therapy on olfactory function. Am J Rhinol Allergy 30:e206-e210, 2016.

19. Kohli P, Schlosser R, Storck K, et al. Olfactory cleft computed tomography analysis and olfaction in chronic rhinosinusitis. Am J Rhinol Allergy 30:402-406, 2016.

20. Massey C, Bury S, Diamond J, et al. Novel use of an ultrasonic bone aspirator for extended endoscopic frontal sinusotomy: A feasibility study. Am J Rhinol Allergy 30:443-447, 2016.

21. Kuan E, Mallen-St. Clair J, Frederick JW, et al. Significance of undissected retromaxillary air cells as a risk factor for revision endoscopic sinus surgery. Am J Rhinol Allergy 30:448-452, 2016.

22. Hong C, Monteiro E, Badhiwala J, et al. Open versus endoscopic septoplasty techniques: A systematic review and meta-analysis. Am J Rhinol Allergy 30:436-442, 2016.

23. Beswick D, Ramadan H, Baroody F, et al. Practice patterns in pediatric chronic rhinosinusitis: A survey of the American Rhinologic Society. Am J Rhinol Allergy 30:418-423, 2016.

24. Adams A, Russell P, Duncavage A, et al. Outcomes of endoscopic repair of cerebrospinal fluid rhinorrhea without lumbar drains. Am J Rhinol Allergy 30:424-429, 2016.

25. D'Anza B, Stokken J, Tien D, et al. Role of lumbar drains in contemporary endonasal skull base surgery: Meta-analysis and systematic review. Am J Rhinol Allergy 30:430-435, 2016. 